\title{
Control of Geometrical Properties of Carbon Nanotube Electrodes Towards High-Performance Microbial Fuel Cells
}

Celal Erbay ${ }^{\mathrm{a}, 1}$, Xiong Pu ${ }^{\mathrm{b}, 1}$, Woongchul Choi ${ }^{\mathrm{b}}$, Mi-Jin Choi ${ }^{\mathrm{a}}$, Yeontack Ryu ${ }^{\mathrm{c}}$, Huijie Hou ${ }^{\mathrm{a}}$, Furong Lin ${ }^{\mathrm{d}}$, Paul de Figueiredo ${ }^{\mathrm{d}, \mathrm{e}, \mathrm{f,g},}$, Choongho $\mathrm{Yu}^{\mathrm{b}, \mathrm{c}, *}$, and Arum $\mathrm{Han}^{\mathrm{a}, \mathrm{h}, * *}$

${ }^{a}$ Department of Electrical and Computer Engineering, Texas A\&M University, College Station, Texas, 77843, USA

${ }^{b}$ Department of Materials Science and Engineering, Texas A\&M University, College Station, Texas, 77843, USA

${ }^{c}$ Department of Mechanical Engineering, Texas A\&M University, College Station, Texas, 77843 , USA

${ }^{d}$ Department of Molecular Pathogenesis and Immunology, Texas A\&M Health Science Center, Bryan, Texas 77807, USA

${ }^{e}$ Department of Veterinary Pathobiology, Texas A\&M University, College Station, Texas, 77843, USA

${ }^{f}$ Norman Borlaug Center, Texas A\&M University, College Station, Texas 77843, USA

${ }^{g}$ Department of Microbial Pathogenesis and Immunology, Texas A\&M Health Science Center, Bryan, Texas 77807, USA

${ }^{h}$ Department of Biomedical Engineering, Texas A\&M University, College Station, Texas 77843, USA

Keywords: microbial fuel cell, carbon nanotube, stainless steel mesh, three dimensional electrode, direct synthesis of carbon nanotube

\section{Corresponding Authors}

* Choongho Yu, E-mail: chyu@tamu.edu Phone: +1-979-862-1073

** Arum Han, E-mail: arum.han@ece.tamu.edu Phone: +1-979-845-9686

${ }^{1}$ Both authors contributed equally to this work. 


\begin{abstract}
In microbial fuel cells (MFCs), physical and electrochemical interactions between microbes and electrode surfaces are critical to performance. Nanomaterial-based electrodes have shown promising performances, however their unique characteristics have not been fully utilized. The developed electrodes here that consist of multi-wall carbon nanotubes (MWCNTs) directly grown in the radial direction from the wires of stainless steel (SS) meshes, providing extremely large three-dimensional surfaces while ensuring minimal ohmic loss between CNTs and SS meshes, fully utilizing the advantages of CNTs. Systematic studies on how different lengths, packing densities, and surface conditions of CNTs affect MFC power output revealed that long and loosely packed CNTs without any amorphous carbon show the highest power production performance. The power density of this anode electrode is 7.4-fold higher compared to bare carbon cloth, which is the highest reported improvement for MFCs with nanomaterial-decorated electrodes. The results of this study offer great potential for advancing the development of microbial electrochemical systems by providing a highly efficient nanomaterial-based electrode that delivers large surface area, high electrochemical activity, and minimum ohmic loss, as well as provide design principles for next-generation nanomaterial-based electrodes that can be broadly applicable for highly efficient microbial electrochemical cells.
\end{abstract}




\section{Introduction}

Microbial fuel cells (MFCs) are sustainable "green" technologies that generate electricity by converting the biochemical energy of microbial metabolism into electrical current $[1,2]$. However the current power performance of MFCs is not sufficiently high to make the technology commercially viable. Maximizing contact and minimizing electron loss between electrochemically active bacteria (EAB) and anodes of MFCs provide routes to improve MFC power performances and lowering the cost of MFCs [3]. A key event occurring at anode-microbe interfaces is electron generation by metabolically and electrochemically active microorganisms [4,5] followed by the transfer of generated electrons to anode surfaces through a combination of direct contact, microbial pili (or nanowires), and/or soluble mediators [6,7]. Regardless of the electron transfer routes, large electrode surface areas promote interactions between microbes (and their corresponding electron shuttle systems) while minimizing the internal electrical resistance.

Carbon cloth is a common electrode material for MFCs because of its relatively low cost and biocompatibility [8]. Three-dimensional (3D) carbon electrodes, in the form of electrospun carbon fiber electrodes [9] or carbon brush [10] electrodes are also used in MFCs, as their 3D structures can provide large surface areas for microbial attachment. However, these conventional electrodes have limited surface areas when compared to nanomaterial-based electrodes. Nanomaterials offer exceptionally large surface-to-volume ratios as well as unique electrochemical properties such as strong charge interactions with organic matter. Recently, various nanomaterials such as metal nanoparticles [11], carbon nanotubes (CNTs) [12-17], and CNT composites $[18,19]$ have been used as additives to MFC anodes. For example, multi-wall (MW) CNTs were attached to carbon cloth, papers, or textiles by using simple dipping methods 
in CNT solutions to increase the electrical conductivity and surface area of electrodes, resulting in $20 \sim 150 \%$ enhancement in power density $[12,15,17]$. The observed improvements in power generation with these electrodes were thought to arise as a consequence of better interactions between EABs and electrodes mainly due to improved electrical conductivity and increased surface areas. Nevertheless, the influence of the physical and geometrical properties of these nanomaterials on power output has not been systematically studied. This analysis is of high importance as nanomaterials can have wide varieties of different physical and geometrical properties depending on their synthesis conditions, including those of CNT-based electrodes.

Notwithstanding large surface-to-volume ratios of CNTs, the aforementioned relatively low power improvement is likely to come from non-optimal use of nanomaterials. For instance, the dipping method makes CNTs lie on the surface of carbon cloth rather than stand erect on this substrate [17]. This configuration significantly impairs the full utilization of large surface-tovolume ratio of CNTs. In cases where CNTs are embedded in polymer composites, CNT surfaces are not fully exposed for charge interactions. These electrode designs also require electrically insulating (or poorly conducting) matrices (e.g., polymer binders) and/or surfactants for CNT dispersions. Thus, electrical contacts between CNTs themselves as well as between CNTs and host electrodes are often poor due to the presence of the intervening insulating organic matter. Here, we attempt to investigate how different lengths, packing densities, and surface conditions of CNTs used as anodes affect MFC power output, as well as propose a CNTdecorated anode configuration that maximizes electron transfer. We expect that this systematic study will result in design principles for developing next-generation nanomaterial electrodes. 


\section{Experimental}

\subsection{Synthesis of CNT electrodes}

MWCNTs were grown on stainless steel (SS) meshes by using a water-assisted chemical vapor deposition (CVD) method. SS 304 and 316 meshes with 400×400 mesh size (opening width 38 $\mu \mathrm{m})$ and $\sim 25 \mu \mathrm{m}$ wire diameter (McMaster-Carr) were used as base electrodes. In order to control the physical properties of CNTs, different catalyst layers were prepared to have five different physical attributes of CNTs (Table 1). For samples S-LD, M-LD, and L-LD, a $10 \mathrm{~nm}$ thick Al film followed by a $\sim 6 \mathrm{~nm}$ Fe film were deposited on SS 304 meshes by using electronbeam deposition. For sample L-HD, the thickness of Fe film was reduced to $\sim 5 \mathrm{~nm}$. For sample M-LD-AC, the surface of SS 316 meshes was roughened by immersing the meshes in $12.4 \mathrm{M}$ $\mathrm{HCl}$ for $30 \mathrm{~min}$. This pretreatment was performed in order to control the morphology of CNTs. For typical CVD processes, the prepared SS meshes (1 inch by 2 inch) were placed at the center of an 1 inch diameter furnace tube. The temperature of a tube furnace was then ramped up to 800 ${ }^{\circ} \mathrm{C}$ in 10 min with 800-sccm dry Ar. The SS mesh was annealed at $800{ }^{\circ} \mathrm{C}$ with 80 -sccm wet Ar and 100-sccm dry Ar for 10, 16, 20, 13, and 5 min respectively for samples S-LD, M-LD, and LLD, L-HD, and M-LD-AC. The wet Ar was prepared by passing pure Ar (99.999\%) through a bubbler containing deionized water. After the annealing process, $200 \mathrm{sccm} \mathrm{H}_{2}(99.999 \%)$ and $100 \mathrm{sccm} \mathrm{C}_{2} \mathrm{H}_{4}(99.999 \%)$ were added to grow CNTs at $800{ }^{\circ} \mathrm{C}$ for $30,30,60,30$, and $60 \mathrm{~min}$ respectively for samples S-LD, M-LD, and L-LD, L-HD, and M-LD-AC.

\subsection{CNT Characterization}

In order to characterize the CNT morphology, scanning electron microscopy (SEMs) were obtained with a JEOL JSM-7500F before and after the MFC runs. In order to inspect microbes with SEMs, pretreatment was conducted. The electrodes were first rinsed with phosphate 
buffered saline (PBS), and then the microbes were immediately fixed using an anaerobic solution of $4 \%$ glutaraldehyde and $4 \%$ formaldehyde for $10 \mathrm{hr}$. Then, the samples were carefully rinsed three times in PBS (pH 7.0) and once in deionized water. Samples were then subjected to a serial dehydration protocol using increasing concentrations of ethanol $(25,50,75,90$, and $100 \mathrm{wt} \% ; 30$ min for each stage with very gentle periodic agitation) and then dried completely at room temperature overnight. Transmission electron microscopy (TEMs) were obtained with a JEOL JEM 2010 to further characterize the surface conditions of CNTs, such as the graphitic layers of CNTs and the amorphous carbon layer (Figure S1).

\subsection{MFC setup and operation}

Microfabricated 24-well MFC arrays (Figure S2) were used for testing the CNT-SS mesh electrodes in parallel. The MFC arrays with 24 independent cells were developed to compare different microbial electrochemical activities. The device was prepared with at least three replicas for each electrode, and their power densities were averaged with standard deviations. The CNT-SS mesh electrodes were punched and attached to gold-deposited anode-side pads using silver epoxy (MG Chemicals). Platinum-loaded carbon paper $\left(0.5 \mathrm{mg} \mathrm{cm}^{-2}\right.$, ElectroChem, Inc) electrodes were also bonded to the cathode-side pads with the same method. Subsequently, the epoxy was cured in an oven for $15 \mathrm{~min}$ at $60^{\circ} \mathrm{C}$ to maximize its conductivity and bond strength. Figure S2a shows a 24-well anode electrode/chamber layer with the CNT-SS mesh electrodes. The cathode chambers were filled with potassium ferricyanide $(100 \mathrm{mM})$ and the anode chambers were inoculated with Shewanella Oneidensis (SO) MR-1 (see the Supporting Information for the preparation). Finally, all components (Figure S2b) were assembled into a system (Figure S2c). All parts were sterilized with 70\% ethanol right before assembling the device. The assembled device was connected to a printed circuit board (PCB) that has a series of 
8 different resistors for each of the 24 units on the device and controlled by multi-position dip switches (Figure S2c). The switch box module was connected to a digital multimeter through a multiplexer (National Instruments) for continuous voltage measurements across the different resistors monitored via a LabView ${ }^{\mathrm{TM}}$ (National Instruments) interface. The measured voltage was converted to current using the Ohm's law, and power output was calculated by multiplying voltage and current. This allowed measurement of power outputs from each of the 24 wells continuously at various load resistances, generating polarization curves for each of the 24 wells as a function of time. Two-chamber MFC was constructed from two $50 \mathrm{ml}$ acrylic chambers separated with a proton exchange membrane (PEM) (Nafion $117^{\mathrm{TM}}$, Ion Power Inc.) that was placed between silicon rubber gaskets to prevent liquid leak (see the Supporting Information for the details).

\subsection{MFC Characterization}

Cyclic voltammetry was carried out to compare the electrochemical activities of the CNT electrodes by measuring current during the oxidation of ferrocyanide. A three-electrode electrochemical cell was prepared with the CNT-SS electrode attached to glassy carbon as a working electrode, a platinum wire as a counter electrode, and an $\mathrm{Ag} / \mathrm{AgCl}$ electrode in $3-\mathrm{M} \mathrm{KCl}$ as a reference electrode. Potassium ferrocyanide $\left(\mathrm{K}_{4} \mathrm{Fe}(\mathrm{CN})_{6}\right)(50 \mathrm{mM})$ was used as an electrolyte at room temperature. The potential on the working electrode was varied from -0.1 to $0.6 \mathrm{~V}$ against the $\mathrm{Ag} / \mathrm{AgCl}$ reference electrode, and current was measured during the potential scanning. The obtained current was converted to current density based on the projected surface area $\left(0.32 \mathrm{~cm}^{2}\right)$ of the working electrode. Chronoamperometry was conducted by applying a potential of $500 \mathrm{mV}$ so as to cause electrochemical oxidation of $\mathrm{Fe}(\mathrm{CN})_{6}{ }^{4-}$. The measurements were performed three times with different pieces obtained from each sample. The active surface 
area of the CNT-SS electrodes was normalized by CNT mass after subtracting the contribution from the SS mesh electrode so as to obtain only CNT characteristics. The uncertainty $(\sigma)$ of all the plots was calculated with each data point $\left(x_{i}\right)$ and mean value $(m)$, and the number of data $(n)$ : $\sigma=\sqrt{\sum\left(x_{i}-m\right)^{2} / n}$

\section{Results and Discussion}

The SS mesh was selected to serves as a backbone of the $3 \mathrm{D}$ electrode material. CNTs grown in the radial direction from the wires of the meshes can provide extremely large surface areas and thus have the potential to maximize contact between EABs and the electrodes, facilitating the transfer of electrons from EABs to the anodes. CNTs were directly synthesized on the SS wires to ensure minimal ohmic loss between CNTs and SS meshes compared to attaching CNTs on electrodes with binders as conventionally done. Table 1 lists five different types of CNT SS mesh electrodes developed in this study. Figure 1a shows a bare SS mesh as a reference. Figure 1b shows CNTs grown on a wire of a SS mesh after scratching off a row of CNTs along the axis of the mesh wire, so as to clearly display excellent physical contacts between the SS mesh and CNTs.

CNTs of different lengths (8, 13, and $19 \mu \mathrm{m}$, Figures 1c, 1d, and 1e, respectively), all being relatively low-density and loosely packed, were synthetized by controlling the catalyst activation time and moisture level. It has been reported that moisture activates the catalyst [20,21], which we found to be the most important parameter in determining CNT growth rates. With a longer activation time, higher growth rates were observed. The growth time with the carbon source were also controlled [22] to vary the CNT lengths. A previously developed 24-well MFC array (Figure S2) having 24 arrays of sub-milliliter anode and cathode chambers and proven to be ideal for 
screening applications [23] was utilized to test the short-term power performances of the developed electrodes. SO MR-1 was used as the EAB and tryptic soy broth (TSB) as substrate for controlled comparison, as wastewater is highly variable, making it not suitable for across-theboard comparison. Comparison of power densities from the electrodes with CNTs of different lengths (Samples S-LD, M-LD, and L-LD) and that from a bare SS mesh (control) showed not only significant improvement, but also significant variations depending on the CNT lengths (Figure 2a). As schematically shown in Figure 2b, with longer CNTs, it is expected that the number of microbes interacting with the CNT electrode increases along the stem of CNTs. Microbial attachment throughout the length of the CNTs like tentacles capturing microbes was confirmed by SEMs (Figure 2c showing side view; Figure 2d showing top view). On the contrary, only a small number of microbes (cylindrical slender objects shown in the inset of Figure 2e) can be laid on the limited two-dimensional wire surface of a bare SS mesh, resulting in low power output. This reflects the significantly higher ( $940 \%$ improvement) power density with the longest CNTs $\left(\sim 19 \mu \mathrm{m}\right.$; sample L-LD; $450 \mathrm{~mW} \mathrm{~m}^{-2}$ at $\left.\sim 3600 \mathrm{~mA} \mathrm{~m}^{-2}\right)$ compared to that of the bare SS mesh control $\left(\sim 0.48 \mathrm{~mW} \mathrm{~m}^{-2}\right)$.

SEM images in Figure 2e, f, g, and h, which respectively depict bare mesh, short, medium and long CNTs (samples S-LD, M-LD, and L-LD) showed significantly more microbes on the electrode with the longest CNTs than their shorter counterparts. This finding correlates well with the significant differences in power densities, as the power density from the long CNTs (L-LD; $\sim 19 \mu \mathrm{m} ; 450 \mathrm{~mW} \mathrm{~m}^{-2}$ ) was $28 \%$ higher than that from the short CNTs (S-LD; $\sim 8 \mu \mathrm{m} ; 350 \mathrm{~mW}$ $\mathrm{m}^{-2}$ ). However the power improvement was limited compared to that from the medium-length CNTs (sample L-LD showing only 5\% higher power than sample M-LD; 13 $\mu \mathrm{m} ; 425 \mathrm{~mW} \mathrm{~m}^{-2}$ ). 
Overall, this result supports the idea that the larger surface areas provided by long CNTs gives higher power output due to the increased numbers of sites for EABs to attach on the electrodes.

Another key aspect influencing the MFC power output is the accessibility of microbes to the CNT surfaces. To investigate this effect, the packing density of CNTs was varied by depositing a thin catalyst layer $(<5 \mathrm{~nm} \mathrm{Fe}$ ) for the densely packed (aligned) CNTs (sample L-HD), compared to a thick catalyst layer (>5 nm Fe) used for the loosely packed CNTs (samples S-LD, M-LD, and L-LD). Since thinner layers often result in smaller and more sites for initiating CNT growth, this resulted in high-density highly aligned CNTs (Figure 1f; L-HD). As schematically shown in Figure 3a, when CNTs are highly aligned and densely packed, the limited space between CNTs is expected to prevent microbes from intercalating into the CNT forest. Indeed, microbes were observed mainly at the tips of the CNTs but not associated with the underlining CNTs (Figure 3b), most likely due to the lack of physical space between the CNTs. The reduced number of microbes, in turn, significantly lowered the maximum power density to $43 \%\left(193 \mathrm{~mW} \mathrm{~m}^{-2}\right.$ for L$\mathrm{HD})$ of that from the low-density CNT electrode $\left(450 \mathrm{~mW} \mathrm{~m}^{-2}\right.$ for L-LD) (Figure 3c). Note that the lengths of CNTs in the low-density CNT electrode (L-LD) and the high-density CNT electrode (L-HD) were similar $(\sim 19 \mu \mathrm{m})$. Mink et al. also used MWCNTs grown through a plasma enhanced CVD method on $\mathrm{Cr} / \mathrm{Ni}$ catalysts. However this approach showed a power improvement of only $26 \%$ compared to bare carbon cloth, probably because microbial growth inside the CNT forest was limited due to the densely packed CNTs and relatively small surface areas due to the large diameters $(200 \sim 400 \mathrm{~nm})$ of the CNTs used in their experiments [13]. The microbial colonization of only the top part of the CNTs does not significantly improve the performance, thus the loosely packed CNTs presented in this work that allow for microbial 
attachment throughout the entire CNTs can contribute to higher power output than densely packed CNTs.

As non-optimal growth of CNTs can easily result in a layer of amorphous carbon on CNTs, we investigated how electrical contacts between microbes and CNTs influence the MFC power output by preparing a CNT electrode having amorphous carbon layers deposited on the graphitic carbon layers of CNTs. The CNT surface condition was altered by etching SS meshes with an aqueous $\mathrm{HCl}$ solution. The etching process roughens the surface of SS meshes, revealing relatively large catalytic Fe sites for CNT growth. This resulted in CNTs covered intentionally with a layer of amorphous carbon (Figure 1g). This thick amorphous carbon coating layer was responsible for the uneven surfaces and larger diameters of CNTs (Figure 1h) as opposed to the other samples (no amorphous carbon layers) displaying smooth surfaces and smaller diameters (inset of Figure 1c). The peak power density of the amorphous carbon-coated CNT electrode (MLD-AC) resulted in $50 \%$ reduction in power to $\sim 210 \mathrm{~mW} \mathrm{~m}^{-2}$, compared to that of similar length and density containing a negligible amount of amorphous carbon (M-LD; $425 \mathrm{~mW} \mathrm{~m}^{-2}$ ) (Figure 4a). A transmission electron micrograph (TEM) of the CNT electrode covered with amorphous carbon shows that a thick carbon layer wrapped around the CNTs (outer walls of CNTs are marked by arrows in Figure 4b). This coating layer made the overall diameter of these CNTs thicker $(100 \mathrm{~nm})$ compared to samples without the amorphous carbon coating $(30 \sim 60 \mathrm{~nm}$; Figure $4 \mathrm{c}$ and Figure S1).

The relatively low power density from the amorphous carbon-coated CNT-SSM electrodes can be ascribed to the amorphous carbon surface covering graphitic layers. The graphitic layer of CNTs often makes relatively strong bonding with organic molecules due to dangling bonds and $\pi-\pi$ interactions [24]. Electrochemically active bacteria can transfer electrons through their pilin 
nanofilaments or microbial nanowires though $\pi-\pi$ interchain stacking and delocalization of charges [25-28], suggesting intimate interactions between microbial nanowires and CNTs. Therefore, the amorphous carbon layer would result in a weaker bonding and thereby a large ohmic loss.

The charge transfer capabilities of the CNT electrodes were further analyzed by performing cyclic voltammetry to obtain potential-current characteristics (Figure 5a). Peak current values $\left(I_{\mathrm{p}}\right)$ were obtained by averaging the results obtained from three different pieces that were cut from each electrode (Figure 5b). All CNT electrodes produced higher current peaks than the bare SS mesh. The long-CNT electrode (L-LD) resulted in the highest oxidation current peak compared to the medium-length and short-length CNT electrodes (M-LD and S-LD). The oxidation slope of the long-CNT was also steep, implying favorable charge transfer. The CNT electrode with amorphous carbon (M-LD-AC) produced relatively low peak current density, likely due to the amorphous carbon layers on the CNTs. Overall, electrodes with higher power densities also showed higher peak current densities. A peculiar trend was observed from the high-density CNT electrode (L-HD), which resulted in a relatively high current density even though the power density was low. This result may come from the large surface area of the densely packed CNTs whose interspaces are large enough for liquid electrolytes to get into the CNT forest but not sufficient for microbes.

The active surface area (A) was also estimated by fitting experimental current data $(I)$ as a function of time $(t)$ at a given voltage to the Cottrell equation:

$I=\left(n F A C_{0} D_{0} D_{0}^{0.5}\right) / \sqrt{\pi t}$

where $\mathrm{n}$, stoichiometric number of electrons involved in the reaction is 1; F, Faraday's constant is $96485 \mathrm{C} \mathrm{mol}^{-1} ; \mathrm{C}_{0}$, concentration of electroactive species is $5 \times 10^{-5} \mathrm{~mol} \mathrm{~cm}^{-3}$; and $\mathrm{D}_{0}$, diffusion 
constant for electroactive species is $8.12 \times 10^{-6} \mathrm{~cm}^{2} \mathrm{~s}^{-1}$. The active surface area $\left(\sim 740 \mathrm{~m}^{2} \mathrm{~g}^{-1}\right)$ of the long, low-density CNT electrode (L-LD) was found to be extremely large (Figure 5c), which was much higher than the geometrically calculated surface area $\left(\sim 50 \mathrm{~m}^{2} \mathrm{~g}^{-1}\right)$ [29] of CNTs whose diameters were $\sim 35 \mathrm{~nm}$. The active surface area displayed a trend similar to the power performance. However, it should be noted that the active surface area was normalized by CNT mass to show electrochemically active surface area per unit CNT mass (i.e., independent of CNT length difference). Interestingly, this plot suggests that the electrochemical reactivity of the CNTs were different from electrodes to electrodes. The CNTs in the low-density CNT electrodes (regardless of their lengths) had larger active areas than those of high-density or amorphouscarbon coated CNT electrodes. The differences in performance between the CNT electrodes with different lengths may be related to the density of CNTs. The density of CNTs near their roots (i.e., SS mesh) was higher due to the growth in the radial direction compared to the density near the CNT tips. When electrochemically active species are oxidized on the surface of CNTs, we believe that they become depleted faster near the root of the CNTs than the tip due to the limited diffusion rate. Since the density of CNTs at the tip is lower than the density near the root because of the radial CNT configuration, the effective electrochemical reactivity of longer CNTs become higher compared to that of shorter CNTs. According to the chronoamperometry results (Figure 5c), the high-density CNT electrode has the smallest active areas, presumably due to the densely packed CNT configuration.

Based on these systematic studies, the CNT electrode having long, loosely-packed, and lowdensity CNTs without any amorphous carbon showed the highest power improvement during the short-term studies. Therefore, this electrode was selected for testing its long-term performance in a two-chamber MFC $(100 \mathrm{~mL})$. The MFC anode chamber $(50 \mathrm{~mL})$ was inoculated with 
anaerobic wastewater and fed with acetate (see Supporting Information for details). After 4-5 days of operation, MFC voltage increased to greater than $0.6 \mathrm{~V}$ (Figure 6a). Polarization curves was obtained after the fresh acetate medium addition, and showed maximum power density of $3360 \mathrm{~mW} \mathrm{~m}{ }^{-2}$. This power density is 7.4-times higher than that from a bare carbon cloth electrode $\left(456 \mathrm{~mW} \mathrm{~m}^{-2}\right)$ (Figure $6 \mathrm{~b}$ ), which is the largest percentage increase with CNT-modified anode reported so far, to the best of our knowledge. The absolute power density from this result is also amongst the highest power densities reported so far (see Table S1 for comparison), however direct comparison of power densities across different MFC platforms and wastewater substrates remains a challenge. Electrochemical impedance spectroscopy (EIS) tests were conducted at open circuit voltage and the Nyquist curves are shown in Figure 6c. The charge transfer resistance of the electrode after microbial biofilm was lower than that of bare carbon cloth, demonstrating that the CNT-grown SS mesh has better performance due to the larger surface area and enhanced biofilm formation which is shown in the SEM image taken after 3 months of MFC operation (Figure 6d).

Overall, the large performance improvement was probably facilitated by the excellent electrical conductivity of CNTs $\left(10^{5} \sim 10^{7} \mathrm{~S} \mathrm{~m}^{-1}\right)$ [30,31], which provides excellent charge pathways with minimal ohmic loss throughout the multiple layers of microbes. For typical twodimensional electrodes, thick biofilms generally contribute to higher electricity generation but their electron transfer efficiency is low due to significant ohmic loss caused by the low electrical conductivity of biofilms (e.g., Geobacter sulfurreducens biofilms was measured to be $\sim 5 \times 10^{-3} \mathrm{~S}$ $\mathrm{cm}^{-1}$ ) [28]. In this case, only a few layers of microbes near electrodes have good charge interactions. Several groups utilized CNT dipping methods, including Xie et al. who fabricated the CNT-textile by dipping a piece of non-conductive textile in CNT ink and improved the 
power density $68 \%$ compared to carbon cloth [15]. The dipping method does not provide strong connection between the CNTs and the base material and thus limits the electron transfer from microbes. Similar methods were also used in several other works $[12,14]$ but the power improvements compared to control electrodes were limited, presumably due to the same reason. The CVD method to directly grow CNTs on the base electrode materials as presented here can increase the efficiency of charge transfer due to the stronger attachment of CNTs. As such, the CVD method used on graphite felt showed 5 times improvement compared to bare electrode [16]. Indeed, the current-voltage sweeping measurements using out electrodes revealed that the contacts between the SS wires and CNTs are ohmic (Figure S3). The stainless steel mesh in our experiment appears to be stable in a short term such as several months. This could be due to direct synthesis of CNTs on SS meshes that coats the meshes with carbon, improving corrosion resistance. However the long-term stability of more than one year for stainless steel still need to be investigated. The use of SS mesh, which can be several times cheaper than the graphite felt, has thus strong potential towards low-cost high-performance MFC anode materials.

\section{Conclusion}

In summary, we synthesized tentacle-like CNTs directly grown from SS meshes in the radial direction of mesh wires, providing microbes with three-dimensional and large surface areas. CNTs captured microbes like tentacles, resulting in excellent charge transfer characteristics presumably due to $\pi-\pi$ stacking between the graphitic carbon rings of CNTs and the pili of microbes. Another key feature of our new electrodes is their minimal ohmic loss due to the direct growth of CNTs from SS meshes. The systematic variation of CNT length, density, and surface condition elucidated the contribution of each parameter to the power performance. It was found 
that the longer CNTs provided more sites for microbes to transfer charge, resulting in higher power output. It was also important to maintain large enough spaces between CNTs for microbes to be intercalated in between. We also found that the graphitic layer of CNTs with minimum amorphous carbon provided an excellent substrate for charge interaction. The CNT-SSM electrode that showed the best performance during short-term testing (long, loosely-packed, and no amorphous carbon) was further tested with wastewater and its long-term performance showed maximum power density of $3360 \mathrm{~mW} \mathrm{~m}{ }^{-2}$, 7.4-times higher than that from carbon cloth, being amongst the highest power improvements in nanomaterial-based MFC anodes. Here, we fabricated a highly efficient nanomaterial-based electrode that offer great potential for advancing the development of microbial electrochemical systems.

\section{Acknowledgements}

This work was supported by the Qatar National Research Foundation (QNRF) grant \#NPRP 5671-2-278 and the Bill \& Melinda Gates Foundation Grand Challenge Exploration Grant (\#OPP1044645). X.P., W.C., Y.R., and C.Y. also acknowledge financial supports from the US National Science Foundation (CMMI \#1030958), and the US Air Force Office of Scientific Research (FA9550-09-1-0609) under the auspices of Dr. Charles Lee for CNT synthesis and characterization. C. E. and X. P. contributed equally to this work. C.E. is supported by the Ministry of National Education of the Republic of Turkey.

\section{References}

[1] B. E. Logan, K. Rabaey, Science 337 (2012) 686-690. 
[2] V.B. Oliveira, M. Simões, L.F. Melo, A.M.F.R. Pinto, Biochem. Eng. J. 73 (2013) 53-64.

[3] J. Wei, P. Liang, X. Huang, Bioresour. Technol. 102 (2011) 9335-9344.

[4] W. Zhi, Z. Ge, Z. He, H. Zhang, Bioresour. Technol. 171 (2014) 461-468.

[5] N. S. Malvankar, D. R. Lovley, ChemSusChem. 5 (2012) 1039-1046.

[6] K. Watanabe, M. Manefield, M. Lee, A. Kouzuma, Curr. Opin. Biotechnol. 20 (2009) $633-641$.

[7] C. I. Torres, A. K. Marcus, H. S. Lee, P. Parameswaran, R. Krajmalnik-Brown, B. E. Rittmann, FEMS Microbiol Rev. 34 (2010) 3-17.

[8] M. Zhou, M. Chi, J. Luo, H. He, T. Jin, J. Power Sources 196 (2011) 4427-4435.

[9] S. L. Chen, H. Q. Hou, F. Harnisch, S. A. Patil, A. A. Carmona-Martinez, S. Agarwal, Y. Y. Zhang, S. Sinha-Ray, A. L. Yarin, A. Greiner, U. Schroder, Energ. Environ. Sci. 4 (2011) $1417-1421$.

[10] A. J. Hutchinson, J. C. Tokash, B. E. Logan, , J. Power Sources 196 (2011) 9213-9219.

[11] Y. Fan, S. Xu, R. Schaller, J. Jiao, F. Chaplen, H. Liu, Biosens. Bioelectron. 26 (2011) 1908-1912.

[12] J. Z. Sun, H.; Yang, Q.; Song, J.; Xue, A., Electrochim. Acta 55 (2010) 3041-3047.

[13] J. E. Mink, J. P. Rojas, B. E. Logan, M. M. Hussain, Nano Lett. 12 (2012) 791-795.

[14] N. Thepsuparungsikul, Phonthamachai, N, Ng, H. Y., Water Sci. Technol. 65 (2012) 1208-1214.

[15] X. Xie, L. Hu, M. Pasta, G. F. Wells, D. Kong, C. S. Criddle, Y. Cui, Nano Lett. 11 (2011) 291-296.

[16] Y. Zhao, K. Watanabe, K. Hashimoto, Phys. Chem. Chem. Phys. 13 (2011) 15016-15021.

[17] H. Y. W. Tsai, C.C.; Lee, C.Y.; Shih, E.P., J. Power Sources 194 (2009) 199-205. 
[18] S. Ci, Wen, Z., Chen, J. \& He, Z., Electrochem. Commun. 14 (2012) 71-74.

[19] T. Sharma, Reddy, A. L. M., Chandra, T. S. \& Ramaprabhu, S., Int. J. Hydrogen Energy 33 (2008) 6749-6754.

[20] K. Hata, D. N. Futaba, K. Mizuno, T. Namai, M. Yumura, S. Iijima, Science 306 (2004) 1362-1364.

[21] P. B. Amama, C. L. Pint, L. McJilton, S. M. Kim, E. A. Stach, P. T. Murray, R. H. Hauge, B. Maruyama, Nano Lett. 9 (2008) 44-49.

[22] D. N. Futaba, K. Hata, T. Yamada, K. Mizuno, M. Yumura, S. Iijima, Phys. Rev. Lett. 95 (2005) 056104.

[23] H. Hou, L. Li, Y. Cho, P. de Figueiredo, A. Han, PloS One 4 (2009) e6570.

[24] D. Tasis, N. Tagmatarchis, A. Bianco, M. Prato, Chem. Rev. 106 (2006) 1105-1136.

[25] K. M. Leung, G. Wanger, M. Y. El-Naggar, Y. Gorby, G. Southam, W. M. Lau, J. Yang, Nano Lett. 13 (2013) 2407-2411.

[26] N. S. Malvankar, M. T. Tuominen, D. R. Lovley, Energ. Environ. Sci. 5 (2012) 62476249.

[27] X. Jiang, J. Hu, L. A. Fitzgerald, J. C. Biffinger, P. Xie, B. R. Ringeisen, C. M. Lieber, Proc. Natl. Acad. Sci. U S A 107 (2010) 16806-16810.

[28] N. S. Malvankar, M. Vargas, K. P. Nevin, A. E. Franks, C. Leang, B.-C. Kim, K. Inoue, T. Mester, S. F. Covalla, J. P. Johnson, V. M. Rotello, M. T. Tuominen, D. R. Lovley, Nat. Nanotech. 6 (2011) 573-579.

[29] A. Peigney, C. Laurent, E. Flahaut, R. R. Bacsa, A. Rousset, Carbon 39 (2001) 507-514.

[30] C. Yu, Y. Ryu, L. Yin, H. Yang, ACS Nano 5 (2011) 1297-1303.

[31] Y. Ryu, L. Yin, C. Yu, J. Mater. Chem. 22 (2012) 6959-6964. 

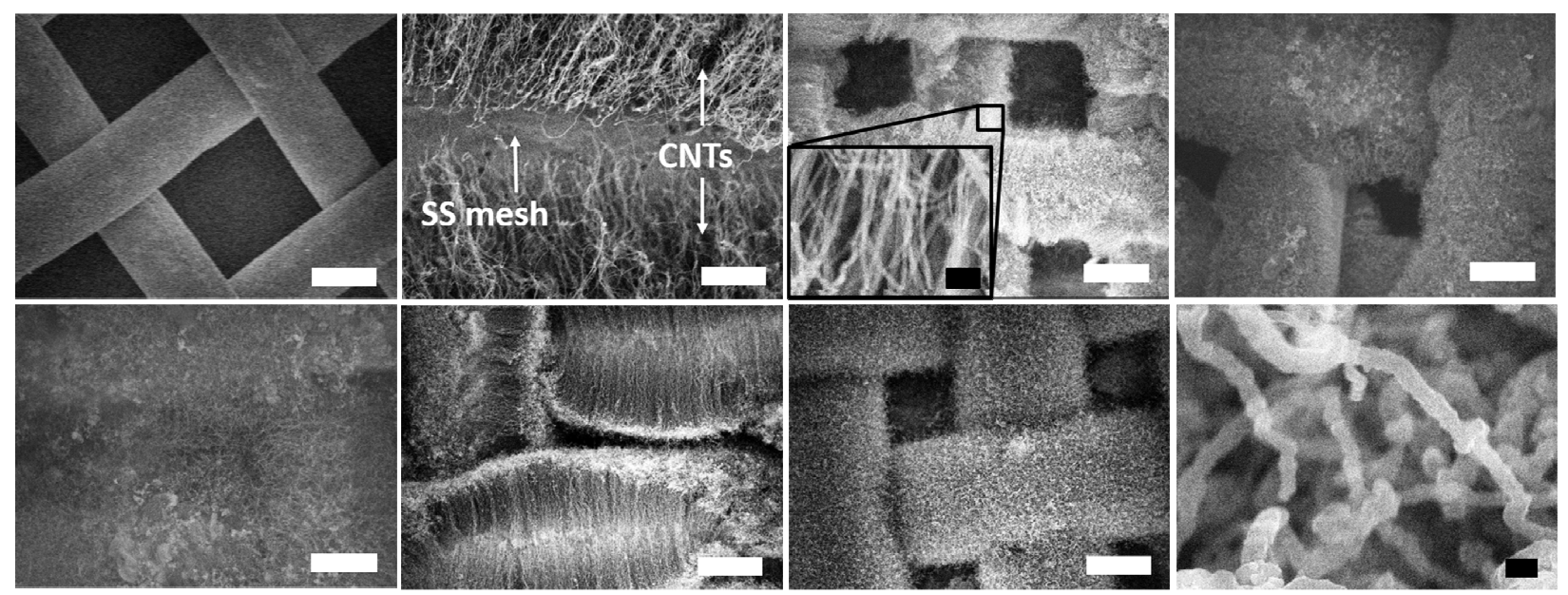

Figure 1. As-synthesized CNT-SS mesh electrodes: CNTs were directly grown in the radial direction from the cylindrical wires of SS meshes. (a) A bare SS mesh without CNTs for reference. (b) CNTs on a SS mesh partially scratched off to expose both the SS mesh and CNTs to show the physical contacts between them. The average lengths of CNTs for S-LD (c), M-LD (d), and L-LD (e) were respectively short ( 8 $\mu \mathrm{m})$, intermediate ( $13 \mu \mathrm{m})$, and long ( $\sim 19 \mu \mathrm{m})$. The length of the CNTs were measured based on the size of the open area between the SS mesh wires (see the decreasing rectangular open areas between the mesh wires in $(\mathrm{c}-\mathrm{e})$ compared to the bare SS mesh in (a). The holes of the SS mesh in L-LD were almost filled by CNTs, compared to $\sim 38-\mu \mathrm{m}$ square opening without CNTs in (a). (f) L-HD showing aligned and densely packed CNTs compared to the other CNTs. (g) M-LD-AC showing amorphous carbon layers intentionally deposited to alter the electrical contacts between CNTs and microbes. (h) The diameter of CNTs with amorphous carbon layers in M-LD-AC is larger than that of S-LD shown in the inset of (c). Scale bars of (a) and (c-g) indicate $20 \mu \mathrm{m}$; (b) $2 \mu \mathrm{m}$; and the inset of (c) and (h) $200 \mathrm{~nm}$. 

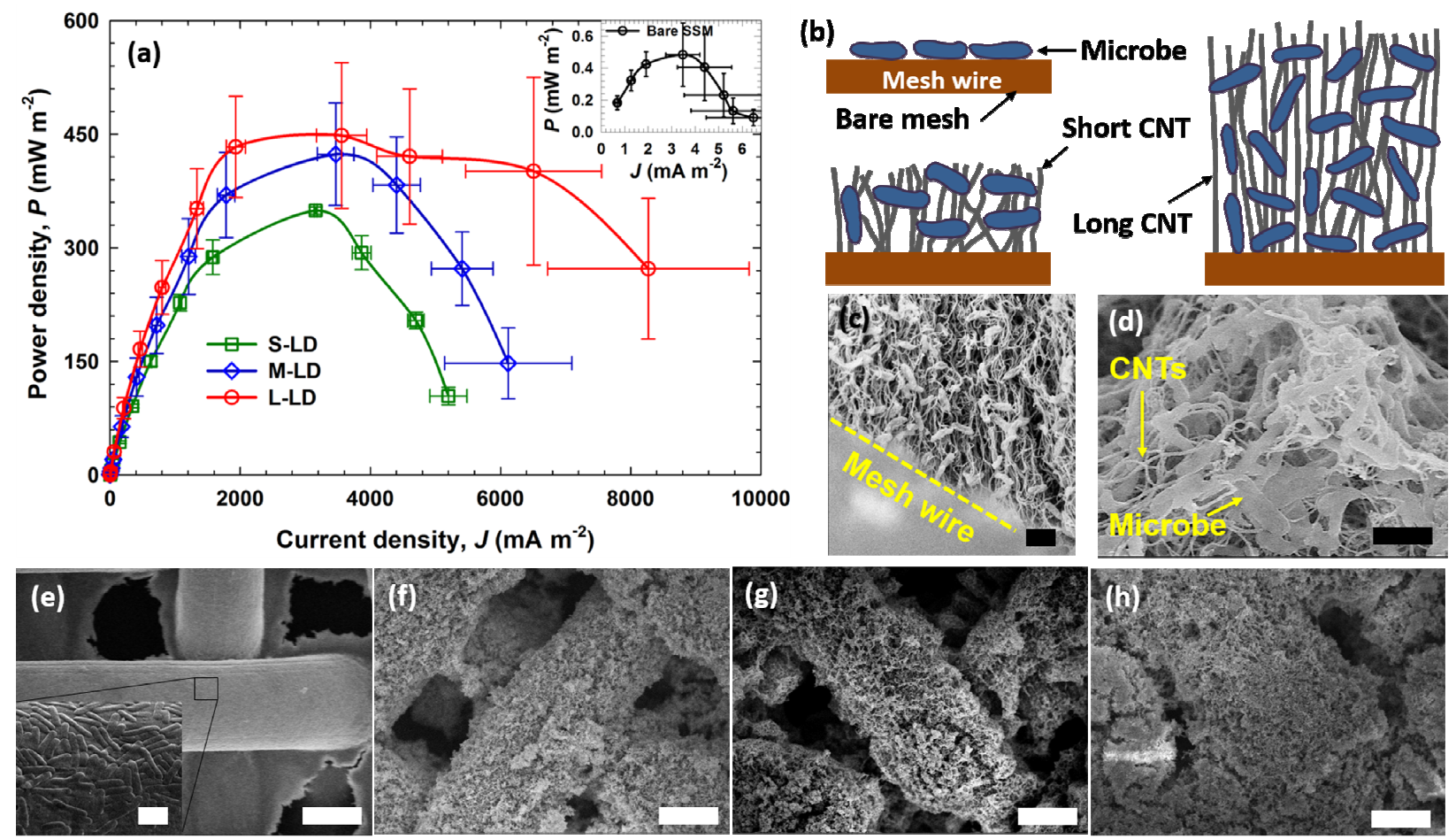

Figure 2. Power performance and CNT-SS mesh electrodes with different CNT lengths after

MFC runs. (a) Power densities against current density of S-LD, M-LD, L-LD, and a bare SS mesh elucidate the effect of the CNT length. (b) Illustration of the interactions between microbes and the CNT-SS mesh electrodes. For a bare mesh, the number of microbes responsible for transferring electrons is limited (upper left pane). Even the electrode with relatively short CNTs (lower left pane) has more microbes than the bare SS mesh electrode. For the electrode with long CNTs, a large number of microbes can interact with CNTs along the stem of CNTs (right pane). This was confirmed by a SEM (c), showing microbes captured by CNTs like tentacles. A row of CNTs were scratched off along the axis of the mesh wire for visualization. An enlarged view from a different angle (d) shows that microbes were entangled in CNTs, providing excellent conduits for transferring electrons. (e) In comparison to the CNT-SS mesh electrodes, a bare mesh with microbes laying on the surface of the mesh wire is shown. S-LD, M-LD, and L-LD after testing are respectively shown in (f), (g), and (h). The scale bars in (c), (d), and the inset of (e) indicate $2 \mu \mathrm{m}$ and those in (e-h) indicate $20 \mu \mathrm{m}$. 

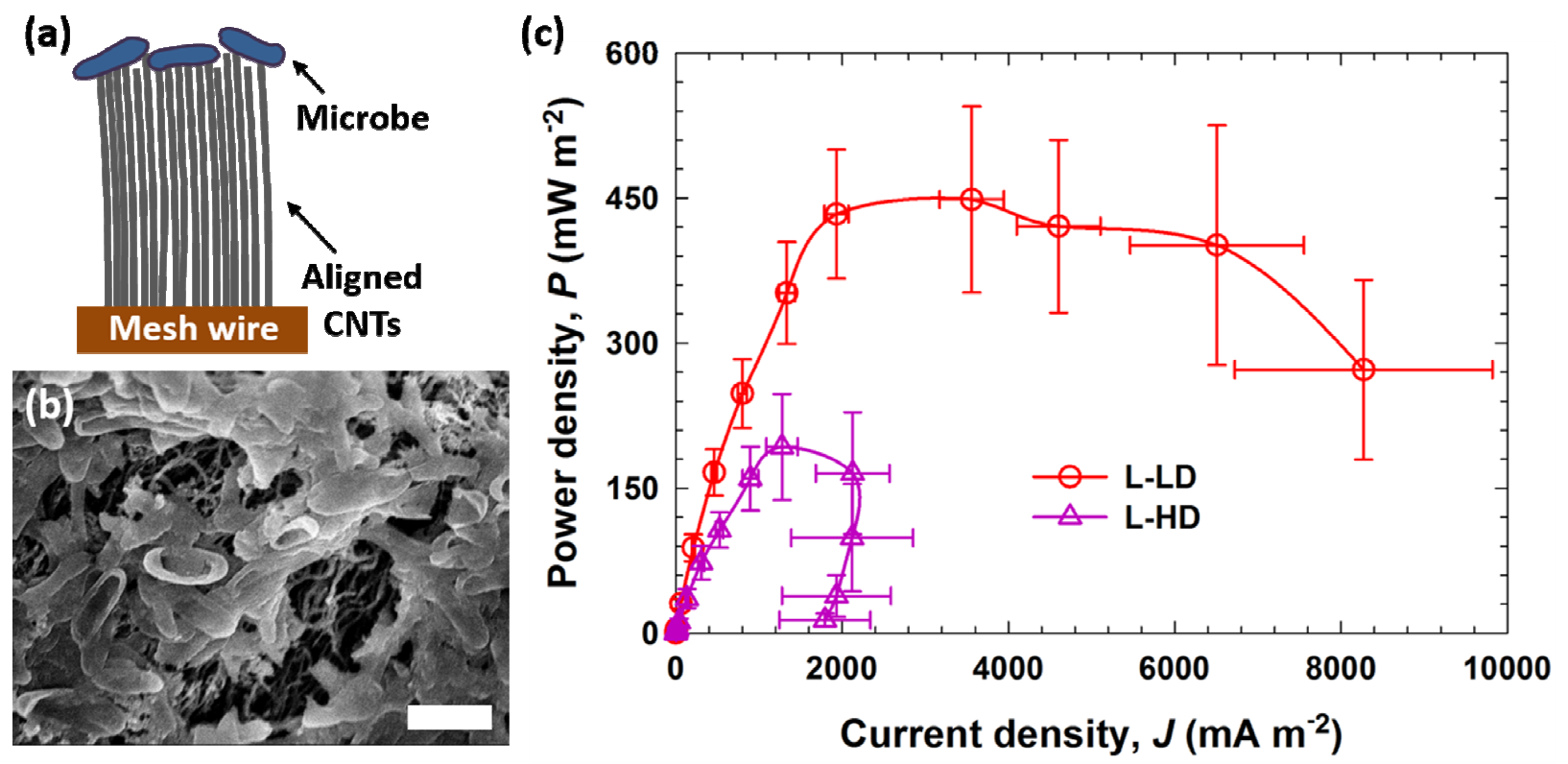

Figure 3. Characteristics of aligned and densely packed CNT-SS mesh electrode. (a) Microbes are difficult to get into the CNT forest, making microbes sit on the tip of CNTs. (b) A top view of L-HD after testing, showing microbes only on the top surface of the CNT electrode but not inside the CNT forest, as can be seen through the cracks of the biofilm. The scale bar indicates 2 $\mu \mathrm{m}$. (c) The power density of L-HD in comparison to that of L-LD whose CNT lengths were similar. 

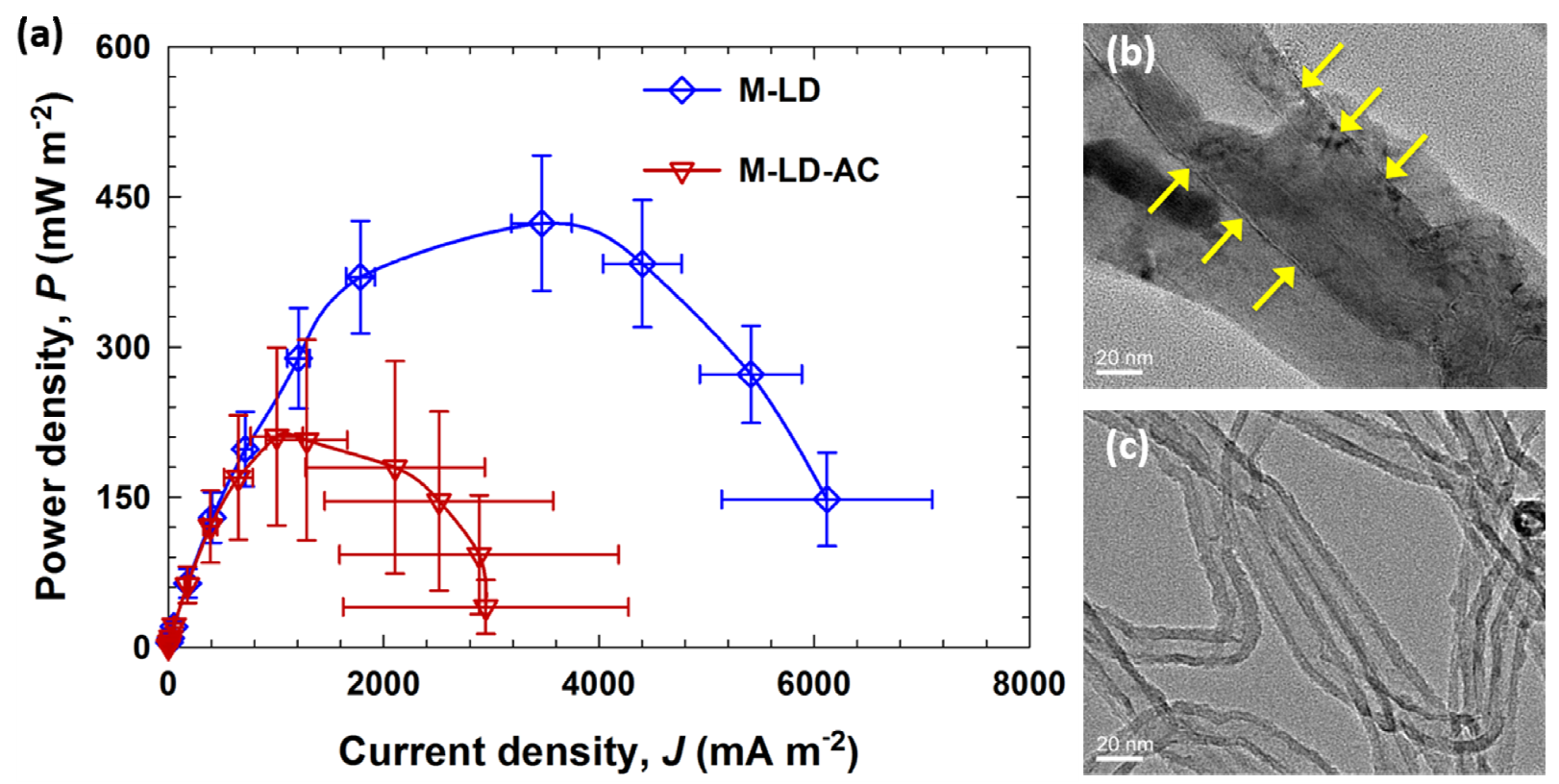

Figure 4. Characteristics of CNT-SS mesh electrode with amorphous carbon coating. (a) The power density of M-LD-AC (amorphous carbon coating layer) in comparison to that of M-LD, whose CNT lengths were similar. (b) Amorphous carbon coating layers were clearly observed from M-LD-AC outside of the CNT. The arrows mark an individual MWCNT. (c) L-LD had only graphitic carbons. 

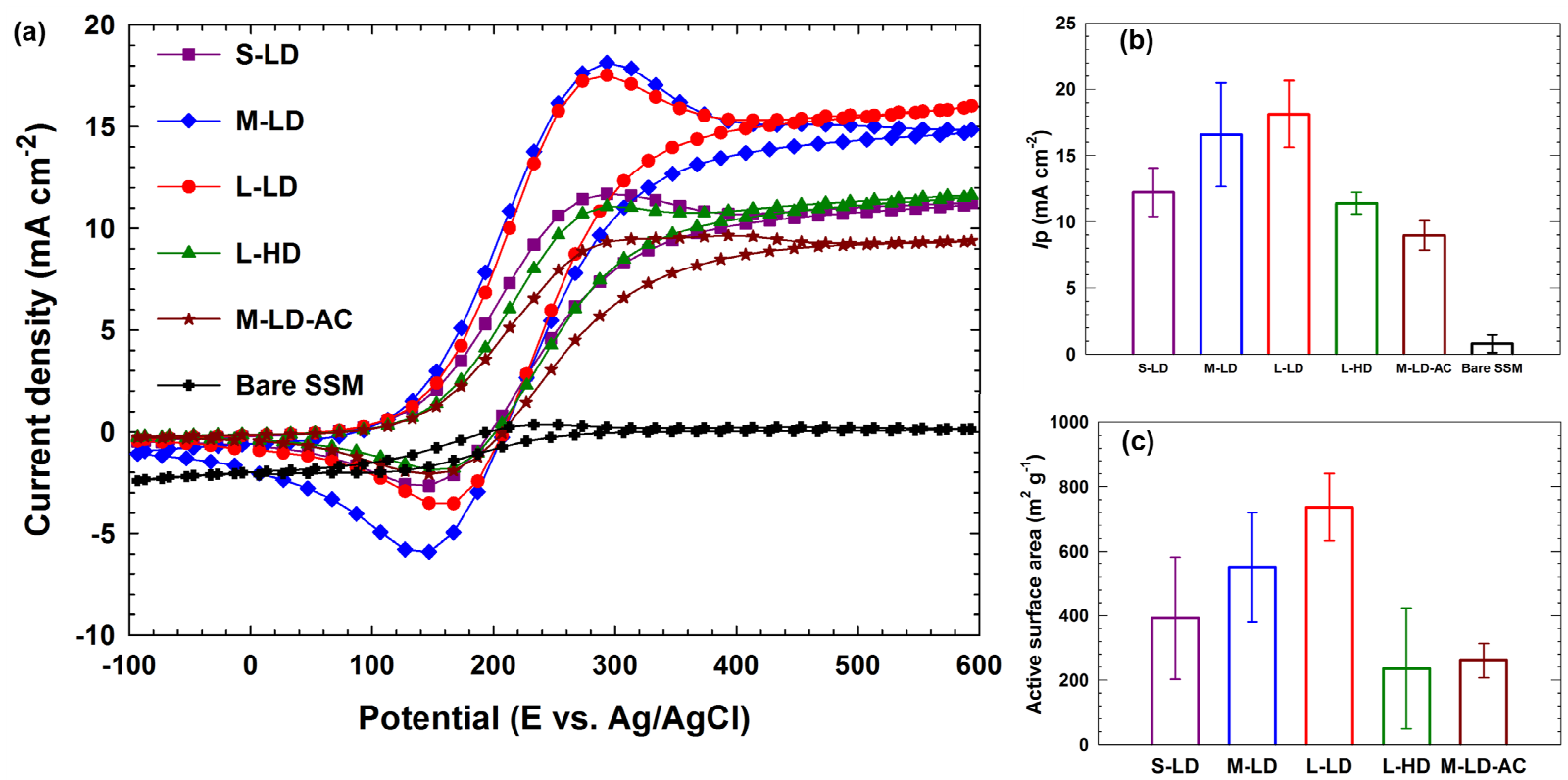

Figure 5. Electrochemical characteristics of CNT-SS mesh electrodes. (a) Representative cyclic voltammetry results of all samples and a bare SS mesh. (b) Peak current densities of all samples and a bare SS mesh in the oxidation cycle. (c) Active surface areas of all samples obtained by performing chronoamperometry. 

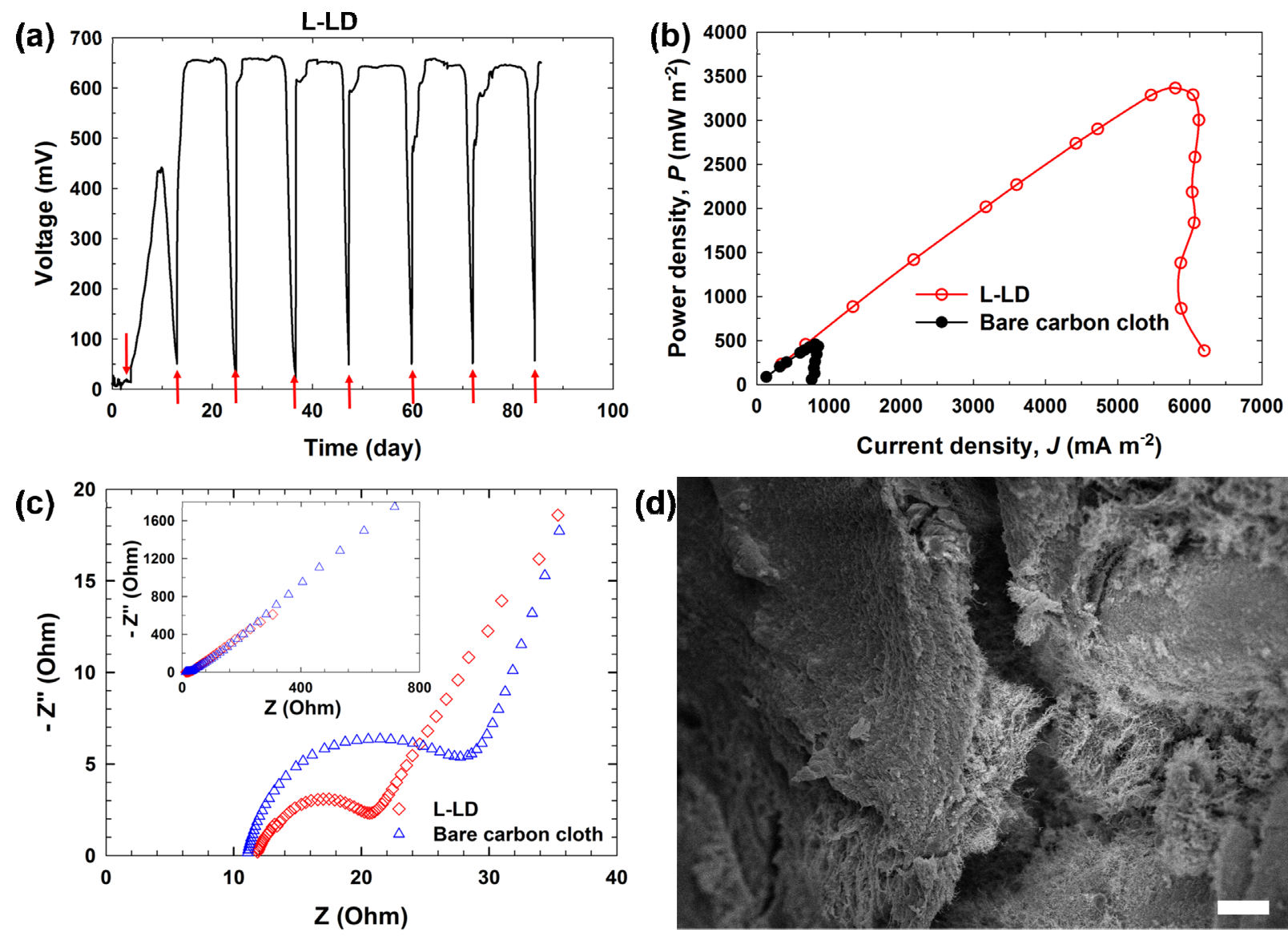

Figure 6. Long-term performance of L-LD in two-chamber MFC device (100 mL). (a) Voltage generation over time showing a sharp increase in voltage after acetate inoculation (arrow marks) and short start-up time (4-5 days). (b) Polarization curves of L-LD and bare carbon cloth (control) showing the maximum power densities achieved with wastewater and acetate and the maximum power density generated by the L-LD anode is 7.4 times that of the bare carbon cloth (3360 $\mathrm{mW} \mathrm{m}^{-2}$ vs $456 \mathrm{~mW} \mathrm{~m}^{-2}$ ). (c) Nyquist curves for the microbial fuel cells equipped with the L-LD and bare carbon cloth. Full curves are shown in an inset graph. (d) SEM image of the microbial growth, cross section of the L-LD after 3 months of operation shows microbial colonization inside of the CNT forest. 
Table 1. A list of CNT-SS mesh electrodes developed to understand how different attributes of CNTs affect MFC power performances.

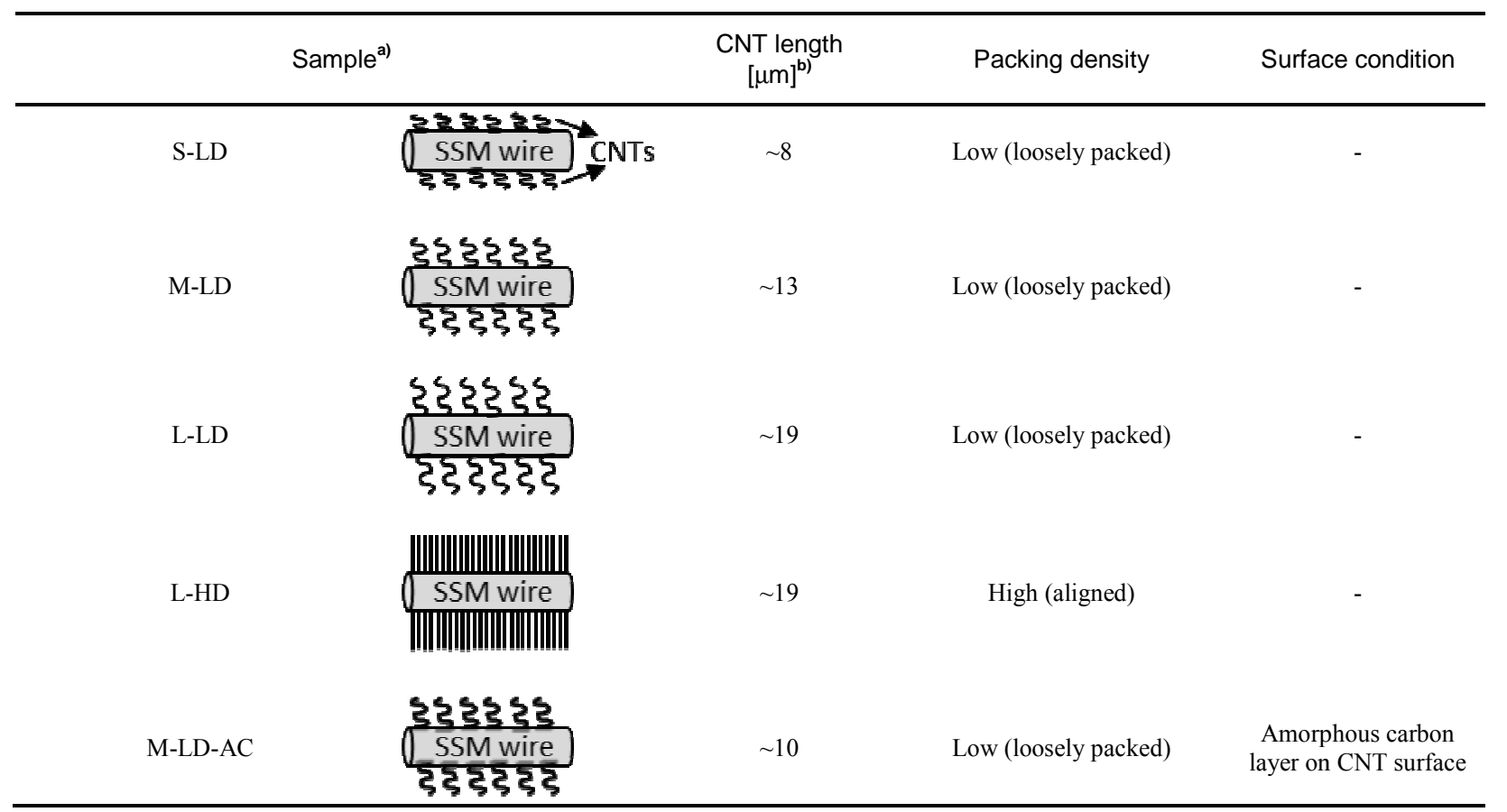

\footnotetext{
a) Sample name nomenclature: (length; $\mathrm{S}=$ short, $\mathrm{M}=$ medium, $\mathrm{L}=$ long) - (density; $\mathrm{LD}=$ low density (loosely packed), $\mathrm{HD}=$ high density and aligned) - (surface condition; none = pure CNT, $\mathrm{AC}=\mathrm{CNTs}$ covered with amorphous carbon); ${ }^{\text {b) }} \mathrm{CNT}$ lengths were estimated by measuring the straight distance from the surface of mesh wires to the tip of CNTs. Actual lengths of the CNTs are likely to be longer than the numbers shown in this table due to the wavy morphology of CNTs.
} 